\title{
Dynamic sizing of energy storage for hedging wind power forecast uncertainty
}

\author{
Pinson, Pierre; Papefthymiou, George; KIöckl, Bernd; Verboomen, Jody
}

Published in:

Proceedings of the IEEE Power Engineering Society General Meeting 2009

Link to article, DOI:

10.1109/PES.2009.5275816

Publication date:

2009

Document Version

Publisher's PDF, also known as Version of record

Link back to DTU Orbit

Citation (APA):

Pinson, P., Papefthymiou, G., Klöckl, B., \& Verboomen, J. (2009). Dynamic sizing of energy storage for hedging wind power forecast uncertainty. In Proceedings of the IEEE Power Engineering Society General Meeting 2009 (pp. 1-8). IEEE. https://doi.org/10.1109/PES.2009.5275816

\section{General rights}

Copyright and moral rights for the publications made accessible in the public portal are retained by the authors and/or other copyright owners and it is a condition of accessing publications that users recognise and abide by the legal requirements associated with these rights.

- Users may download and print one copy of any publication from the public portal for the purpose of private study or research.

- You may not further distribute the material or use it for any profit-making activity or commercial gain

- You may freely distribute the URL identifying the publication in the public portal 


\title{
Dynamic Sizing of Energy Storage for Hedging Wind Power Forecast Uncertainty
}

\author{
Pierre Pinson, George Papaefthymiou, Member, IEEE, Bernd Klöckl, Member, IEEE, and Jody Verboomen, Member, IEEE
}

\begin{abstract}
In market conditions where program responsible parties are penalized for deviations from proposed bids, energy storage can be used for compensating the energy imbalances induced by limited predictability of wind power. The energy storage capacity necessary for performing this task will differ between delivery periods, according to the magnitude and the evolution of forecast errors in each delivery period. A methodology is presented for the assessment of the necessary storage capacity for each delivery period, based on the degree of risk that the power producer accepts to be exposed to. This approach leads to a dynamic assessment of the energy storage capacity for different delivery periods. In such a context, energy storage is used as a means of risk hedging against penalties from the regulation market. The application of the algorithm on real data (both measurements and forecasts) of the yearly output of a wind farm shows that the application of a dynamic daily sizing of the necessary storage leads to a significant reduction of the storage capacity used, without affecting the producer's profit significantly. The method proposed here may provide the basis for the introduction of storage as an independent market entity, where each producer may rent the necessary daily storage capacity for hedging the risk of the wind power limited predictability.
\end{abstract}

Index Terms-wind power, storage, electricity markets, forecast, uncertainty, scenarios

\section{INTRODUCTION}

The increased participation of wind power in electricity markets leads to the management of energy portfolios under higher uncertainty due to the limited predictability of wind power production for the time-scales involved. Information about the expected performance of state-of-the-art prediction methods is available in e.g. [1]. When participating in electricity pools, this uncertainty is translated into revenue loss for the wind power producer, induced by regulation costs. A possible countermeasure for compensating these losses is to use electrical energy storage (EES) as a buffer for absorbing the imbalances, by storing the surplus of energy and using it in periods of deficit. The issue of optimal sizing, optimal operation, or value of storage to be used in combination with wind power generation has inspired a number of recent studies in the literature, e.g. [2]-[7].

The key issue for deciding on an optimal operation of a combined wind/EES plant, or for the sizing of the storage device itself, is to have access to a realistic representation of wind power forecast uncertainty. In a general manner, such representation must include information on the probabilistic distribution of potential generation for each lookahead time, as well as information on the correlation structure of

G. Papaefthymiou and J. Verboomen have been partly supported by SenterNovem (an agency of the Dutch ministry of Economic Affairs) within the framework of the research program 'Intelligent Power Systems'. P. Pinson has been partly supported by the European Commission through the project ANEMOS.plus (EU FP6, contract $\mathrm{n}^{\circ}$ 038692).

P. Pinson is with the Technical University of Denmark, DTU Informatics, Kgs. Lyngby, Denmark (e-mail: pp@imm.dtu.dk).

G. Papaefthymiou is with Electric Power Systems, Delft University of Technnology, The Netherlands, and with Ecofys Germany GmbH, Berlin, Germany (email: g.papaefthymiou@ecofys.com).

B. Klöckl is with Verbund APG, Vienna, Austria (email: bernd.kloeckl@ verbund.at).

J. Verboomen is with Siemens AG, Erlangen, Germany (email: jody.verboomen@siemens.com). At the time this work was carried out he was with Electric Power Systems, Delft University of Technnology, The Netherlands,

978-1-4244-4241-6/09/\$25.00 C2009 IEEE forecast errors. This last point is unfortunately often not appropriately considered in studies focusing on wind/ESS operation and sizing, e.g. [6]. In parallel, assumptions on probabilistic distributions of potential wind power generation (like Gaussianity for instance in [4]) are highly unrealistic even though attractive in terms of reducing problem complexity. Not appropriately accounting for those aspects may lead to erroneous results, and underestimation of necessary storage capacity.

In this paper, statistical scenario forecasts of short-term wind power production, representing both the potential deviations between forecasts and actual power production, and the correlation pattern of prediction errors, are used as a basis. Those scenario forecasts are generated following the method recently described in [8]. A generic methodology is presented for deciding upon the energy storage capacity that may be needed for hedging operational uncertainty induced by the wind power forecasts employed. Such hedging strategy involves a risk parameter $\alpha$, corresponding to the risk for the combined wind/EES system of not being able to fulfill the contract made on the day-ahead market. The central idea is to dynamically allocate the necessary storage capacity. Based on the scenario forecasts, the power producer can decide upon the energy storage capacity necessary for the following delivery period, leading to an overall reduction of the needed storage, without severe impact on the revenues. Simplifications are made here about the storage model employed, or about the market information that would be used in real-world application, since we only aim at presenting the core of the methodology. This methodology is indeed generic: the dynamic storage assessment corresponds to the first application from a series of different tasks in the energy portfolio management that may be redefined accordingly, like reserve allocation, unit commitment, etc.

In a first stage, the basics concerning imbalance management in a market environment are discussed in Section II. The role of the EES is then investigated in Section III. In Section IV the methodology for the dynamic sizing assessment of the EES is presented, followed by an example application for the operation of a wind farm in the case study of Section V. The paper ends with concluding remarks in Section VI, along with perspectives for future work.

\section{IMBALANCE MANAGEMENT IN A MARKET ENVIRONMENT}

In deregulated electricity markets (e.g. the Scandinavian Nord Pool market or the Dutch power exchange APX) power producers dispatch their production by proposing quantity-price energy bids in advance in the day-ahead (spot) market over the following delivery period for each unit of time (Program Time Unit, abbreviated PTU). In view of the time-scales involved, a market participant with a portfolio including a significant share of wind power must base his bid on wind power production forecasts with horizons ranging from 13 to 37 hours ahead. It is known that forecasts for such look-ahead time have a highly variable level of accuracy, which is also quite low on average, see [9], [10]. Since market participants are charged for any deviation from contract, information on the situation-dependent uncertainty of the forecasts provided prove to be paramount. The bids on the dayahead market are all matched through a single auction process for determining the market clearing price (spot price) and the program 
of the participants for each PTU. The real-time balance between generation and load is performed through the regulation market, managed by the Transmission System Operator (TSO). Regulation of positive and negative imbalances translates to penalties for the market participants, defined as the volume of electricity in imbalance times the imbalance price. These imbalance prices are different for positive and negative imbalances, and highly variable, being a function of the power volume that needs to be regulated globally in the system, supposed to reflect the production (or curtailment) costs, and including a premium for readiness [11]. As a consequence in such market environment, the profit of the power producer is defined as the revenue obtained from the day-ahead market minus the penalties resulting from imbalance regulation costs (see revenue formulation in e.g. [12]).

In order to maximize his profits, the power producer should then minimize the imbalance costs by more efficient bidding consisting of minimizing the cost of power imbalances. These efficient bidding strategies should be based on wind power forecasts and information on their uncertainty, along with information on regulation unit costs [12]-[14]. When the energy portfolio consists of multiple units, the power producer can minimize the power imbalances in each PTU by considering curtailment of the wind power production (or reduction of the output of conventional generation means) in the case of surplus, or inversely use conventional units as backup on idle or on reduced power in the case of deficiency. This however unavoidably leads to lower unit utilization, lower efficiencies and consequently higher emissions. Another alternative here is the use of large-scale EES as a buffer for absorbing the fluctuations around the delivery contract proposed on the day-ahead market, by storing surplus of energy and using it in periods of deficit. The key question here is the operation, size and type of EES necessary for performing this task.

\section{ROLE OF EES: OPERATION, TYPES AND SIZING ISSUES}

\section{A. Basic EES modeling principles}

The combined wind/EES power plant is presented in Fig. 1. The wind power production of the wind power plant is denoted as $P_{v}$. A part of this power can be curtailed $\left(P_{c}\right)$ or be used for charging the EES $\left(P_{c h}\right)$. Furthermore, additional power can be delivered by the discharging of the EES $\left(P_{d c h}\right)$. The output power of the combined plant $P_{o}$ can thus be written by the power balance equation ${ }^{1}$,

$$
P_{o}=P_{v}-P_{c}-P_{E E S},
$$

with the terminal power of the EES being denoted as $P_{E E S}$. The energy content of the EES (denoted as $E$ ) generally evolves as a function of the terminal power and a loss function due to

$$
\dot{E}=P_{E E S}(t)-P_{L}\left(P_{E E S}, E, t\right),
$$

where in the time discrete case, the above equation reads

$$
\frac{\Delta E}{\Delta t}=P_{E E S}-P_{L}\left(P_{E E S}, E, \Delta t\right) .
$$

The constraints related to the operation and simulation of the combined wind/EES power plant consist of the output power limits, the physical limits to energy storage operation (maximum and minimum energy contents), and numerical limits related to the finite time step $\Delta t$ in a time discrete simulation. For a detailed discussion on this topic, see [15].

\footnotetext{
${ }^{1}$ Reactive power is neglected in this analysis, but can easily be incorporated when considering possible ancillary services delivered by the combined plant.
}

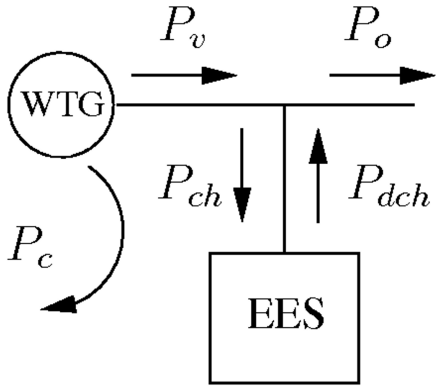

Fig. 1. The combined wind/EES power plant.

\section{B. EES operation under different market conditions}

There may be different views of what the optimal operation of a wind/ESS power plant in a market environment is. If having a system point of view, one may be concerned with maximizing the overall penetration of wind power [2], or one may alternatively account for constraints related to frequency/voltage issues [7]. If being a participant in a deregulated electricity market with the only aim of benefit maximization, one may envisage that storage will be used for arbitrage, i.e. for taking advantage of the daily variations in the spot market prices by shifting quantities of energy from low price periods to high price periods [16], [17]. In this context, the operational strategy may be obtained as an internal part of a larger linear optimization algorithm, where the objective function is the maximization of the daily profit by shifting power generation between different time periods.

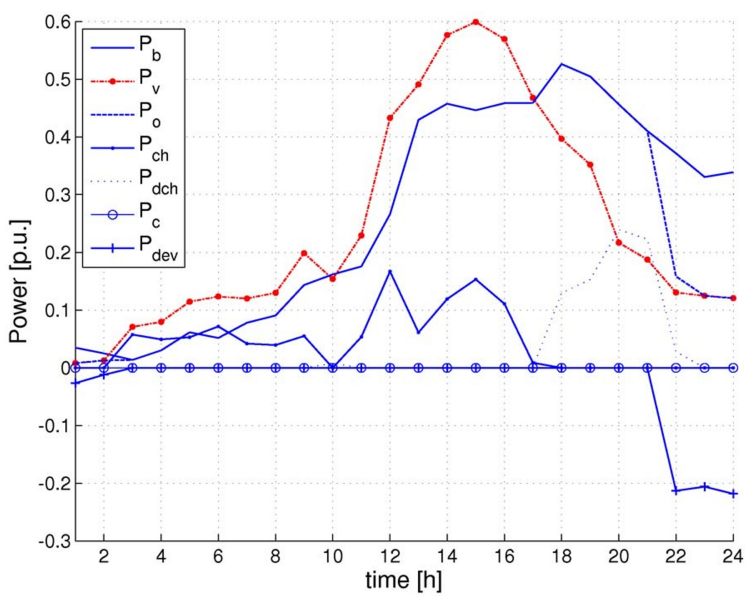

Fig. 2. Daily operation of the combined wind/EES power plant.

However, in electricity markets where wind power producers have to pay for their deviations from contract, one may then consider that the EES operation is actually determined by the sign of the imbalances and the energy content limits. I.e., when the imbalance is positive and there is spare capacity, the EES is charged and when negative and there is spare energy content in the EES, the EES is discharged. In such a case, this market participant is trying to maximize his revenue by minimizing the potential regulation costs induced by the deviations from contract. This is the situation we concentrate on in the present paper. Fig. 2 presents the operation of the combined wind/EES plant in such a market environment when the curtailment option is not present. It is observed that the EES manages to fix the power output to the bidding schedule $\left(P_{b}\right)$ up to hour 21, when the EES is empty and negative imbalances continue. One can also notice that the storage actually acts as an integrator over 
the energy imbalances for consequent PTUs under the objective of minimizing the regulation costs. In such a context, the main influence on the EES operational strategy derives solely from the choice of the bidding strategy. This strategy should be determined as an integral part of a general stochastic optimization where the EES operation is incorporated in the trading principles introduced in [12].

\section{The EES sizing issue: principles of dynamic sizing}

As mentioned above, the EES operates as an integrator over the energy imbalances. Therefore, the EES capacity that is necessary for absorbing the energy fluctuations is determined from both the magnitude and the sequence of the imbalances. Consequent imbalances of different signs are counterbalanced by the EES without the need for extra capacity. To illustrate this, an example of the operation of an EES device for two different imbalance patterns is presented in Fig. 3. In both cases, the energy deviations take the same values for the total period ( 5 occurrences of +1 p.u. and 5 occurrences of -1 p.u.) but in the two cases the sequence of the events is different. In the first case all deviations with the same sign occur consecutively, while in the second the sign of the deviations is changed in each PTU. As can be easily observed, the sequence of deviations has a major effect on the necessary EES capacity; in the first case a capacity of 5 p.u. is necessary, while in the second 1 p.u. is enough.

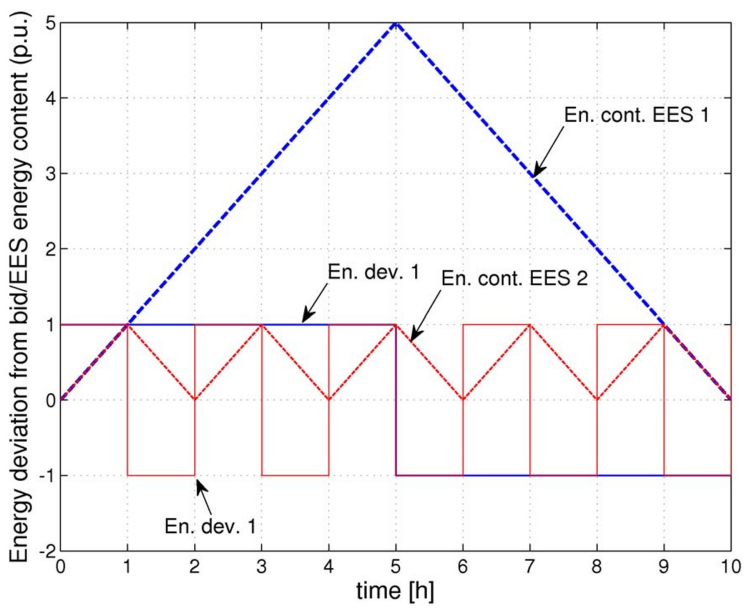

Fig. 3. Example of EES operation for 2 different sequences of imbalances.

This simple example shows how one may calculate the necessary storage capacity when the actual energy production for each PTU is known. However, at the time of bidding this information is not available. Instead, the power producer may possess information on the forecast uncertainty. Using this information, it can be possible to assess a set of scenarios corresponding to all possible futures for the wind power development. Based on these scenarios, the necessary storage capacity may be evaluated. Each scenario however will yield a different energy capacity. The set of possible energy capacities will correspond to a probability distribution which may be used by the energy producer to decide upon the necessary storage capacity. Since the forecast uncertainty varies between different delivery periods, the necessary capacity will not be fixed, but will deviate from one period to the other. Therefore, if the power producer may assess the forecast uncertainty in a form of possible wind power scenarios for the next delivery period, then it is possible to decide upon the optimal storage size necessary for minimizing imbalances.

The assessment of forecast uncertainty will play a central role for the definition of the EES capacities. As presented in the example in Fig. 3, one should accurately model both the forecast error magnitude and temporal development through the interdependence structure of forecast errors. Considering independent normal distributions for the modeling of forecast errors for the different horizons ahead as in [16], [18] for instance is not appropriate. Such approaches do not take into account neither the probabilistic information on the forecast uncertainty nor the interdependence structure of forecast errors with a given forecast series. In the present paper, a method recently presented by the authors in [8] is used, allowing for the generation of scenario forecasts of wind power production that comply with both requirements.

\section{Methodology FOR EES DYNAMIC SIZING}

\section{A. Scenario forecasts of wind power production}

Owing to the limited predictability of wind generation and the highly variable level of accuracy of point forecasts of wind power, information about the situation-dependent uncertainty of such forecasts is paramount [10]. The most promising manner of doing so is to generate probabilistic forecasts of wind power, either from purely statistical methods (see e.g. [19]), or by conversion of ensemble forecasts of meteorological variables to ensemble forecasts of wind power [20], [21]. Probabilistic forecasts give the complete probability distribution of potential wind power production for each look-ahead time, commonly up to $48-72$ hours ahead.

A drawback of probabilistic forecasts is that they are produced independently for each look-ahead time. Thus, they do not inform on the development of the prediction errors through prediction series, since they neglect their interdependence structure. As illustrated and explained above, this information is of particular importance for many time-dependent and multistage decision-making processes, such as the operation of a wind/EES system. In order to satisfy this additional requirement, one needs to generate scenarios of short-term wind power production. This can be done with the method described in [8]. The resulting scenario forecasts respect the (nonparametric) probabilistic forecasts for the next period, and also rely on the most recent information about the interdependence structure of prediction errors. Monte-Carlo simulation is further used for the generation of the scenarios. The problem is defined as the sampling from a multidimensional distribution with arbitrary marginals and can be treated using the techniques presented in [22], based on the transformation of the marginals between different domains. First a multivariate Normal distribution is used for generating a set of Gaussian variables realizations that are correlated according to the estimated interdependence structure and then these Gaussian variables realizations are transformed to the desired marginals. Fig. 4 depicts an example with the commonly provided point forecasts of wind generation, corresponding probabilistic forecasts, as well as a set of 25 scenarios forecasts for a period of 43 hours ahead. This example is for the case of a multi-MW wind farm in Denmark.

\section{B. EES dynamic sizing}

Based on the above methodology, a number of scenario forecasts are generated, corresponding to the possible futures of the wind power production. Since the methodology for generation of scenario forecasts is not computationally expensive, a large number of scenarios can be generated and subsequently employed for decision-making. For each of these scenarios, the combined wind/EES plant operation can be assessed. This operation is defined by the bidding strategy, the storage size, the charging/discharging power limits and the EES energy content in the beginning of the delivery period.

Let us assume that a market participant directly bids the provided point forecasts of wind generation on the day-ahead market. In Fig. 5, the result of the possible scenarios for the EES energy content for 


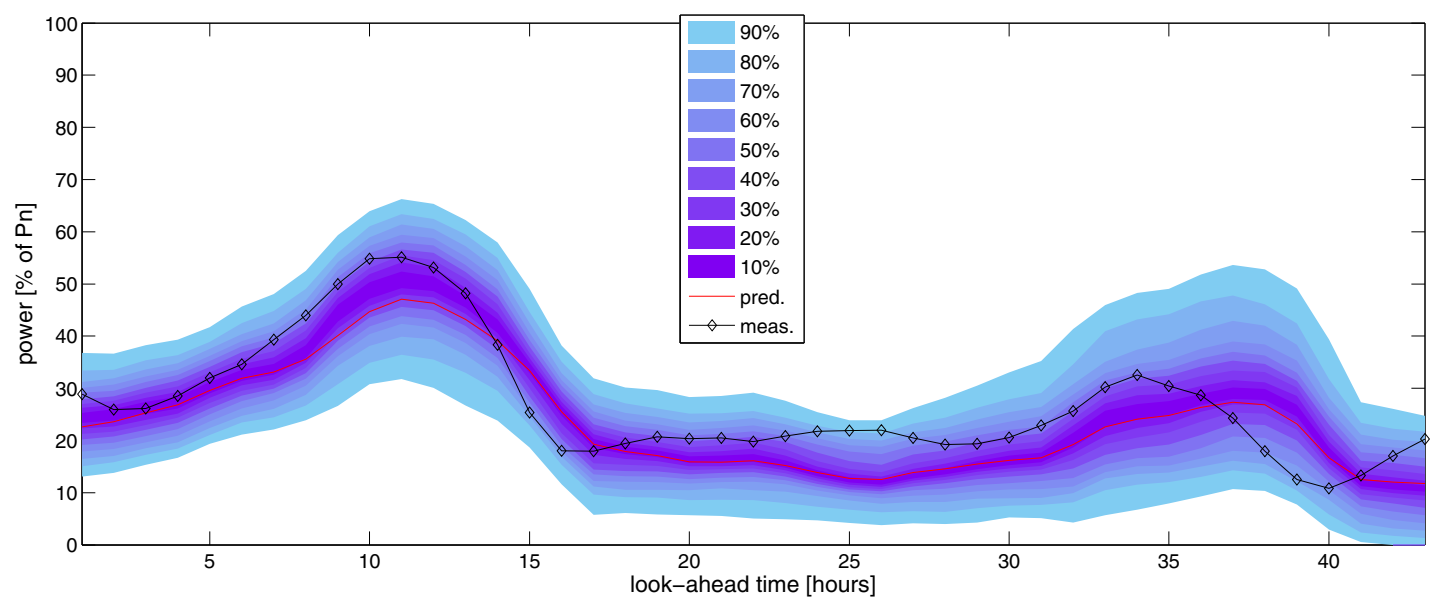

(a) Point and probabilistic forecasts

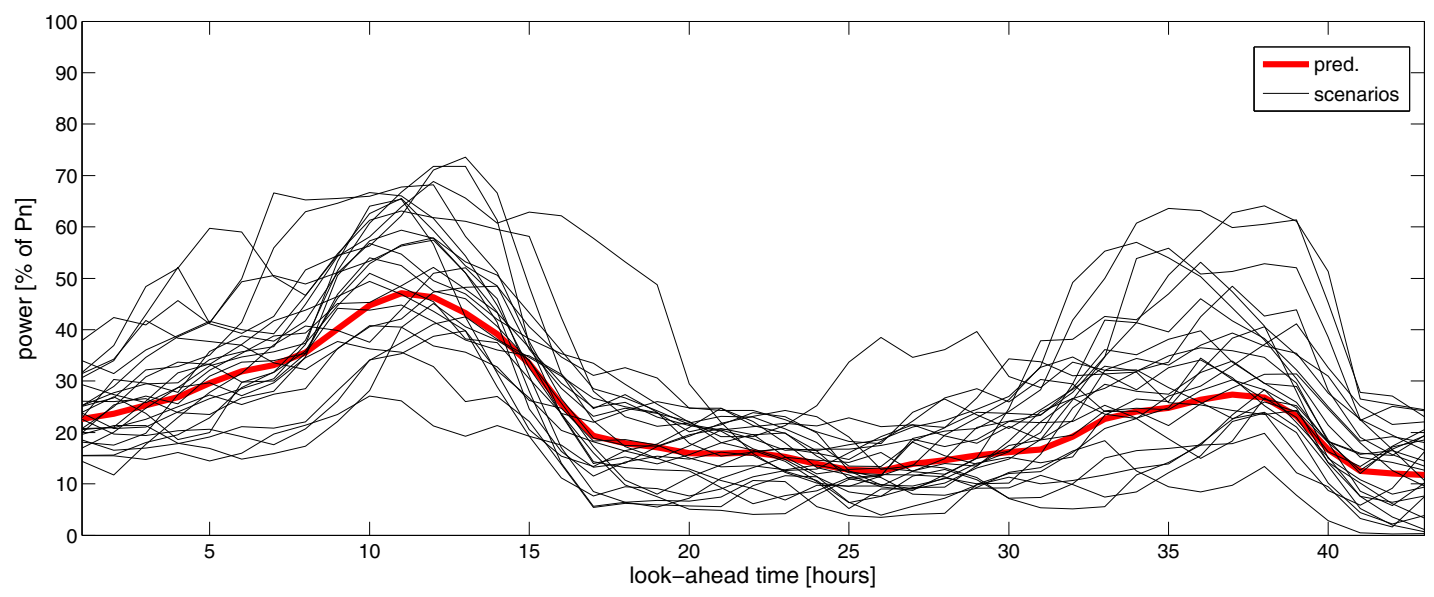

(b) Corresponding scenario forecasts

Fig. 4. Point and probabilistic forecasts of wind generation, along with a set of 25 scenario forecasts, for a multi-MW wind farm in Denmark.

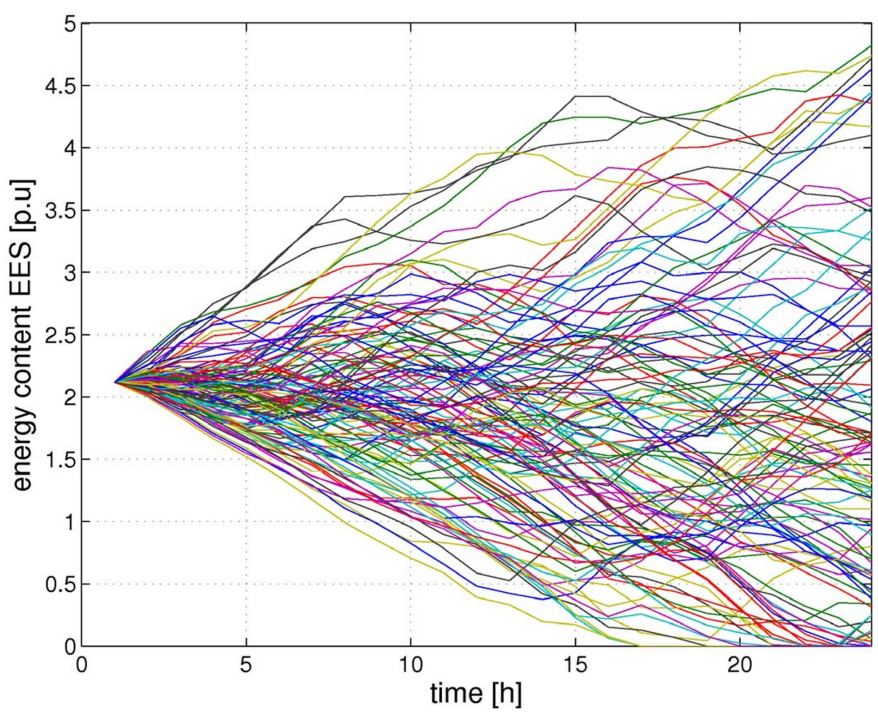

(a) Time plot

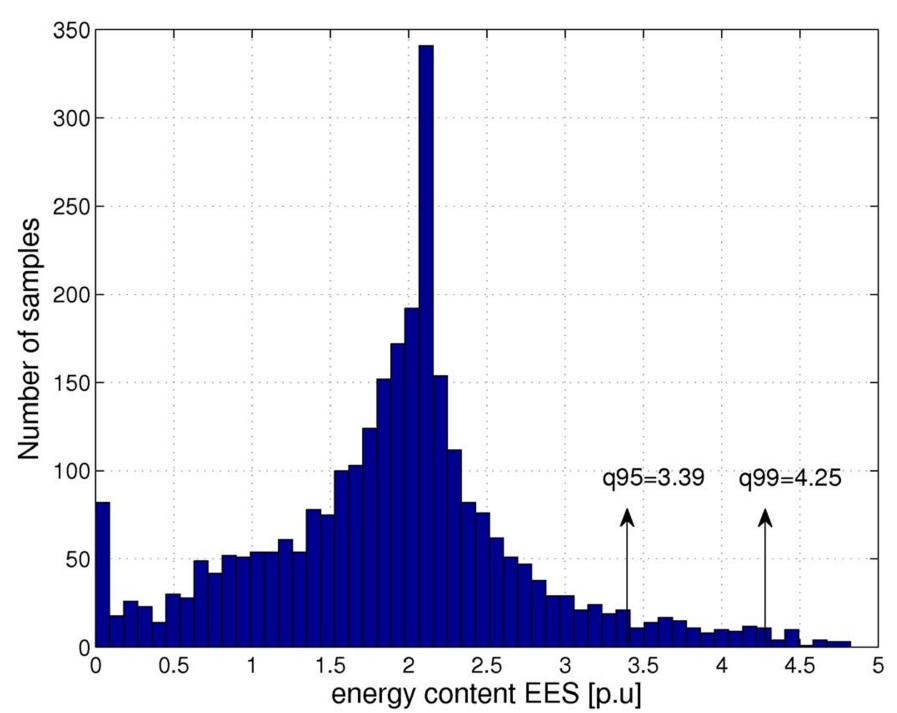

(b) Histogram

Fig. 5. Assessment of the day-ahead potentially necessary EES energy contents.

the day-ahead operation of the wind/EES power plant is shown. The different scenario forecasts lead to different daily operation 
of the EES, thus to different potentially required EES capacities. By gathering the information in a histogram, one may estimate the probability distribution of the necessary EES energy content. For instance in Fig. 5(b) is given the energy content distribution corresponding to the EES operation of Fig. 5(a). For this specific case, it appears that with an EES capacity of 4.82 p.u. the market participant producer can be $100 \%$ sure that the power imbalances for the next delivery period are minimized. A lower EES capacity would translate into a risk of not having enough EES capacity to fulfill this task. This risk can be quantified by a parameter $\alpha$, meaning that there is a risk of $\alpha \%$ of not covering potential imbalances with the storage device. Inversely, this risk can be quantified if deciding on a certain storage capacity. In Fig. 5(b) we can see the EES capacity that corresponds to different quantiles of the distribution, namely the 95\% and $99 \%$ quantiles, corresponding to risk parameter values of $\alpha=5 \%$ and $\alpha=1 \%$, respectively.

In this point lies the core of the present contribution. Using the information on the wind power uncertainty for the next delivery period, the power producer may decide upon the necessary EES capacity based on the level of risk exposure, translated into a specific quantile of this distribution. For example, if a market participant decides upon a risk hedging of $95 \%$ (thus for $\alpha=5 \%$ ), the necessary energy content will correspond to the $95 \%$ quantile of the energy content distribution (Fig. 5(b)). Since the forecast uncertainty will differ among different delivery periods, so will the necessary storage capacity. Using the proposed technique, the power producer may dynamically allocate the necessary storage capacity based on the desired risk exposure. In such a context, storage could be envisaged as a service that could be contracted by the market participant for hedging his portfolio against regulation costs.

\section{CAse STudy}

The case of a Danish multi-MW wind farm is used as a basis. Point forecasts result from the application of the Wind Power Prediction Tool (WPPT) method [23], which uses meteorological predictions of wind speed and direction as input, as well as historical measurements of power production. All power predictions and measures, which are normalized by the wind farm nominal capacity $P_{n}$, cover a period of one year. Hourly updated point predictions have an hourly resolution (similar to measurements) up to 43 hours ahead. Then, nonparametric probabilistic forecasts are produced with time-adaptive quantile regression [19]. Predictive distributions are given by 19 quantile forecasts whose nominal proportions range from 0.05 to 0.95 with a 0.05 increment. From a probabilistic point of view, since it is not possible to exclude any possibility, even though extreme prediction errors might happen, the very tails of predictive distributions are modeled as exponential tails. An evaluation of the quality of probabilistic forecasts produced with this method is given in [24]. Fig. 4(a) gives an example of such probabilistic forecasts of wind generation, in the form of a fan chart. In parallel, Fig. 4(b) depicts a set of 25 scenarios of power production for the same period, generated from the method of [8] (briefly introduced in Section IV-A), along with the traditionally provided point forecasts for the coming period. For the study performed in the following, the number of scenario forecasts to be generated for each period is set to 120 . Remember that this number can easily be raised to several thousands for better uncertainty quantification.

The operation of a wind-hydro generation facility is investigated, consisting of a hydroelectric power plant with an electric generator, a wind park, lower and upper water reservoirs and penstock and pumping pipes. The energetic round-trip efficiency of the EES, corresponding to the global hydraulic circuit efficiency is assumed to be $\eta_{s}=\eta_{c h} \cdot \eta_{d c h}=75 \%$ without contribution from standby losses.
The charging and discharging efficiencies are further considered as equal, $\eta_{c h}=\eta_{d c h}$, and the function $P_{L}$ from equation 2 is assumed to be of first order ${ }^{2}$. Both maximum hydro generation and pump nominal capacity are considered as $25 \%$ of the wind park installed capacity. The cost of charging the EES $\left(c_{c h}\right)$ corresponds to the internal cost of the pumping operation and is taken as equal to $1.5 € / \mathrm{MWh}^{3}$.

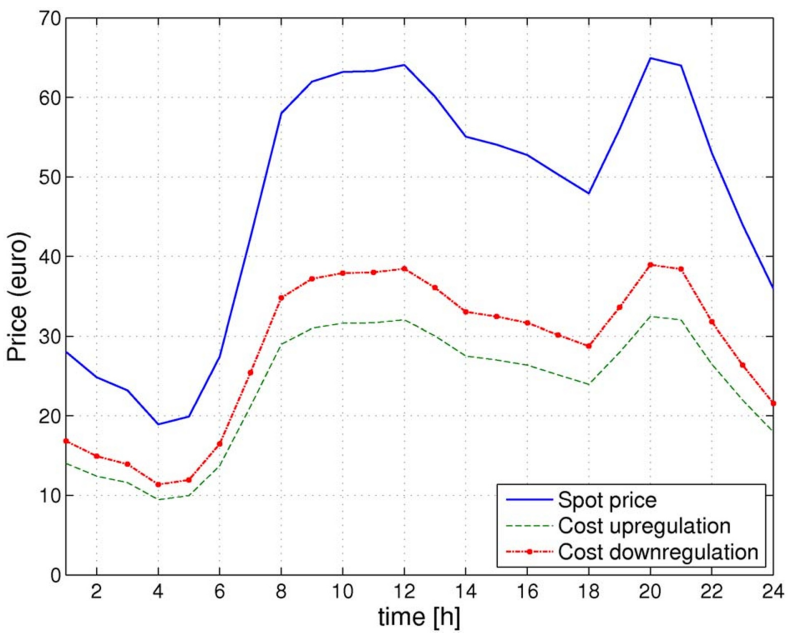

Fig. 6. Profile of spot prices and regulation costs used in the study, based on yearly averages.

The size of the EES is defined dynamically based on the principles presented in Section IV-B. Possible remaining energy in the reservoir after each delivery period ( 1 day) is transferred to the next one. The operation of the combined wind/hydro plant for a total period of 1 year is simulated. The combined plant is considered to operate in a typical electricity pool. It is considered as a first simple case that the market participant does not have access to advanced price forecasts, and makes decisions based on climatologital forecasts corresponding to the average over the year. The corresponding spot prices and unit regulation costs used for the evaluation of the market operation are depicted in Fig. 6. The unit cost for upregulation is considered as $50 \%$ of the spot price, while that for downregulation amounts to $60 \%$ of the spot price. These values are inspired by the analysis of the Nord Pool market data performed in [25]. Note that this study may be easily extended to incorporate better information on prices. Also, the price uncertainty may be included in a second step in the decision making-process, so that the decision on the dynamic sizing derives from an overall regulation cost hedging point of view. Here, prices are not used for decision-making, but more for illustrating how the dynamic sizing of energy storage may impact the regulation costs for a given auction period or over a period of time.

In Fig. 7(a) the dynamic EES capacity assessment based on the 99\% quantile (risk parameter $\alpha=1 \%$ ) is presented. In particular, for each day, an EES capacity is defined based on the $99 \%$ quantile of the respective energy content distribution (see Fig. 5(b)). As expected, the size of the necessary EES varies throughout the year, based on the anticipated wind power forecast uncertainty. Using this EES capacity for each day of operation, the system operation has been simulated. In Fig. 7(b), the hourly time-series of actual energy content of the dynamically sized EES is depicted over this same year of operation. One may then observe how this time-series are correlated with the

${ }^{2}$ It has been shown in [15] that constant efficiency assumptions are of limited practicability in realistic investigations, but will be used here for the sake of simplicity. More advanced loss mechanisms may be included in the methodology and is the subject for future work.

${ }^{3}$ These settings for the hydro facility are obtained by related literature [16]. 


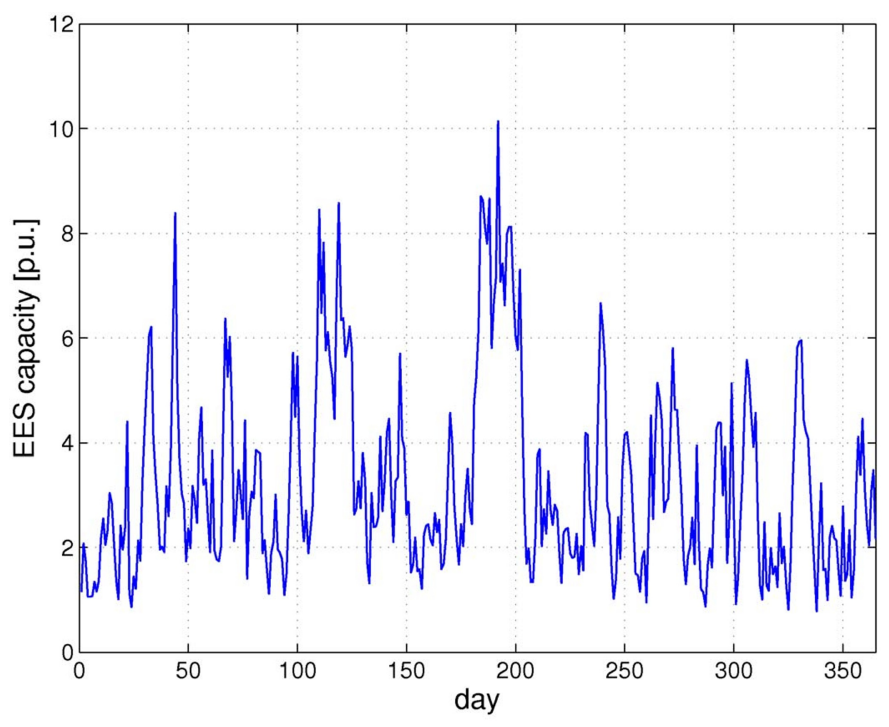

(a) EES Capacity

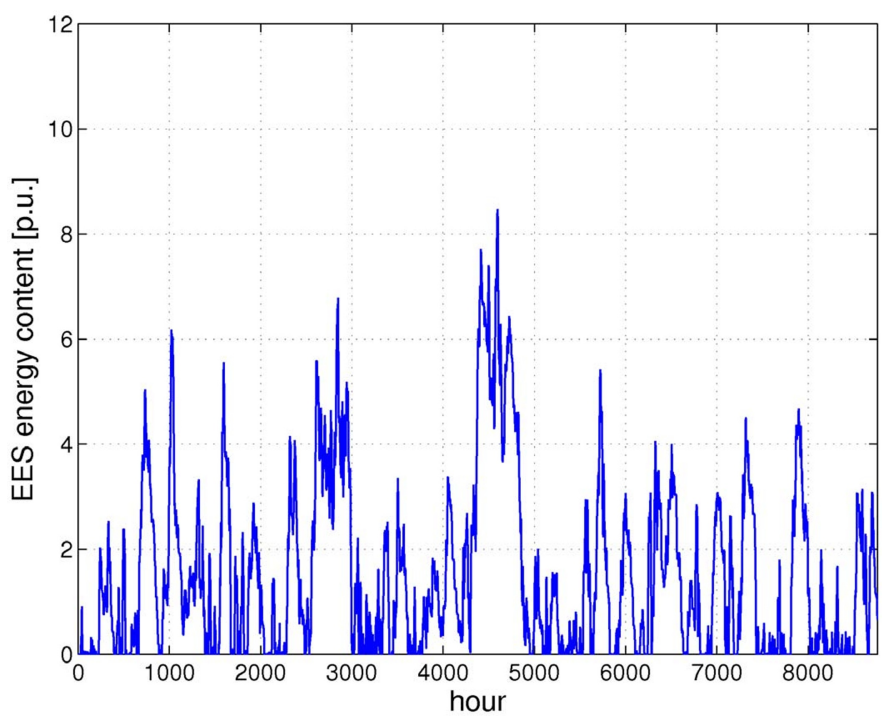

(b) EES Energy Content

Fig. 7. EES dynamic capacity assessment based on the $99 \%$ quantile (risk parameter $\alpha=1 \%$ ) and estimated energy content (1 year of operation).

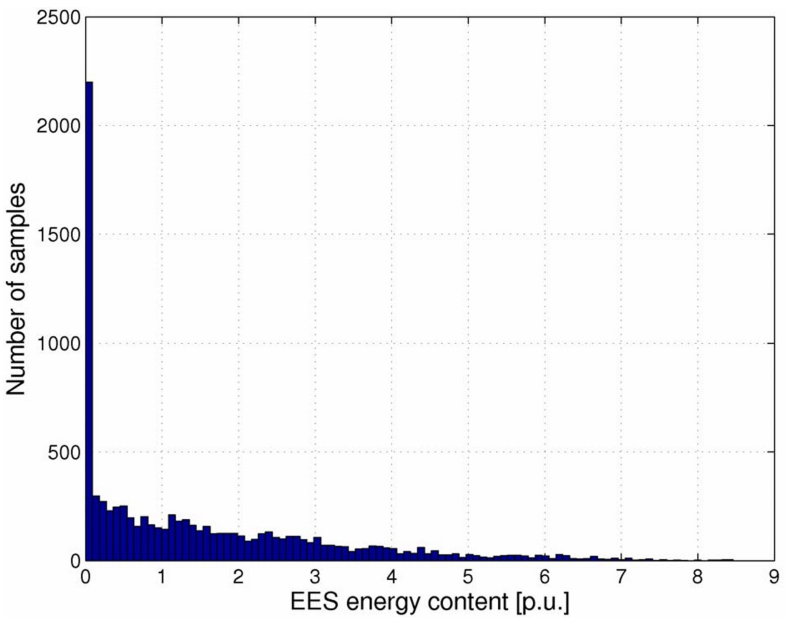

Fig. 8. Histogram of the EES estimated energy content for one year of operation.

daily sizing of the EES. In parallel, Fig 8 summarizes the histogram of the energy content for 1 year of operation corresponding to Fig. 7(b). For a large fraction of time, the energy storage is empty. There are two reasons for that. On the one hand, the fact the storage is appropriately dynamically sized does not mean that it has the optimal amount of energy stored at the beginning of the delivery period. For instance, if the methodology for a given day tells there is a need for large storage capacity, but the storage is empty at the beginning of the delivery period, there will be not possibility of compensating for negative imbalances. On the other hand, the effect of the EES losses is quite dramatic. Indeed, since the power bid corresponds to the point forecast, the positive and negative imbalances are expected to be asymptotically equal. Therefore, one should expect the EES to operate as a buffer that levels out these imbalances. However, losses make that the EES can only release $\eta_{s} \%$ of a positive imbalance previously stored. This lead to a frequent emptying of the storage device. The amount of energy lost due to the EES losses is a critical parameter for the assessment of storage as discussed in detail in [15]. Therefore ideally, a market participant using a wind/EES should slightly reduce his bid from the wind power point forecasts in order to ensure there will be more energy stored than released, which in terms of positive and negative imbalances would result in even volumes.

In order to assess the economic feasibility of the methodology, the operation of the combined wind/EES plant for the 1 year is simulated, for 20 possibilities for EES sizing: (1) no storage, (219) dynamic EES sizing based on the 17 quantiles rising from $15 \%$ to $95 \%$, thus for risk parameters going from $5 \%$ till $85 \%$, with $5 \%$ increment, and the $99 \%$ quantile $(\alpha=1 \%$, very low risk exposure), (20) infinite storage ${ }^{4}$. Each of these scenarios corresponds to a different strategy that the wind producer may follow for hedging wind power forecast uncertainty. In Fig. 9(a), the total profit for the producer and the regulation costs for each scenario are presented. Following the proposed methodology, the dynamic sizing strategy according to a low risk exposure (low $\alpha$ ) ensures a profit that is very close to the case of a dedicated infinite storage. As shown in Fig. 9(a), employing higher values of the risk parameter $\alpha$ directly lead to higher risk exposure, and resulting costs on imbalances, finally yielding a reduced overall revenue. For risk parameter values between $65 \%$ and $85 \%$ (thus relying on quantiles with proportions between $15 \%$ and $35 \%$ quantile) the storage size is so small that it actually does not offer any profit. In Fig. 9(b) the respective maximum and mean EES capacity for the operation of the system for each scenario is presented, calculated by the respective EES capacity for each day (see e.g. Fig. 7(a) for the $99 \%$ quantile scenario). One can see that the maximum and mean EES capacity needed is reduced with the decreasing quantiles, meaning that higher risk exposure, leads to a reduced need for EES capacity.

\section{Vi. CONCLUSIONS - FUtURE WORK}

In market conditions that do not favor wind power against other energy sources, electrical energy storage can be used for minimizing the imbalance costs due to the limited predictability of wind power. The storage size necessary for performing this task depends on the forecast error for each delivery period. Since this error varies between

\footnotetext{
${ }^{4}$ As infinite storage capacity the minimum capacity for hedging all wind power forecast errors is considered, quantified based on the analysis presented in Section IV-B as 12.08 p.u. Any storage capacity larger than this will be underused.
} 


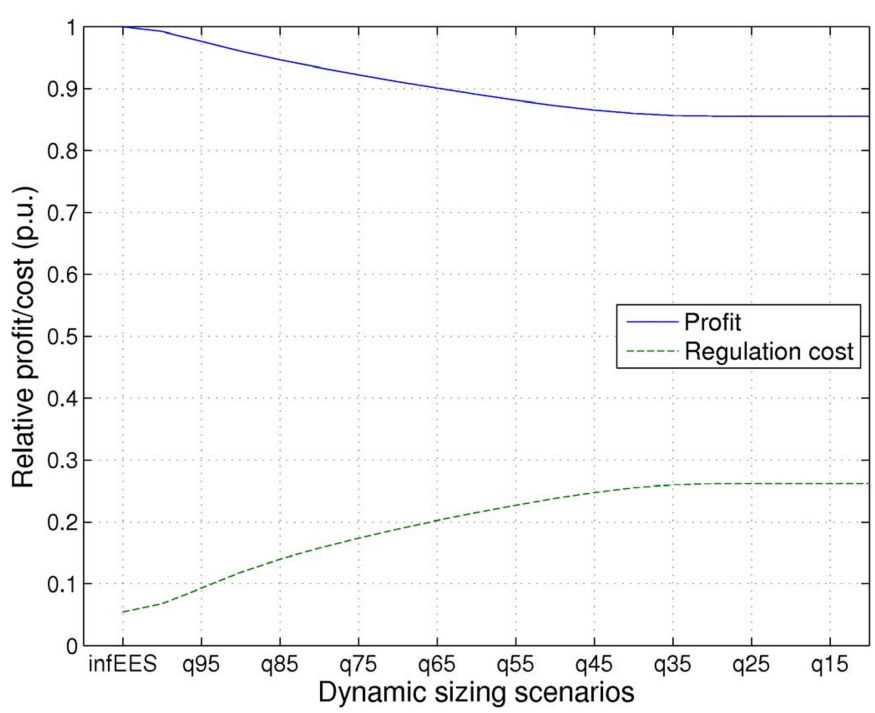

(a) Profit/cost curves

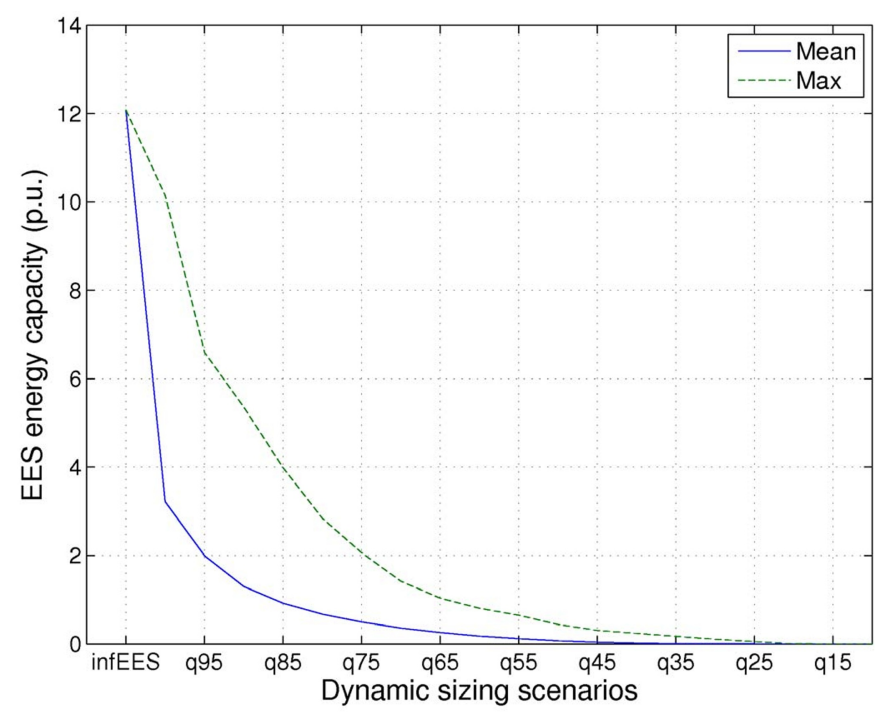

(b) EES max/mean Energy Content

Fig. 9. Profit/cost curves and EES max/mean energy content for the different dynamic sizing scenarios.

successive delivery periods, the necessary storage is also expected to follow these variations. Therefore, the assignment of dedicated storage based on a worst-case scenario (e.g. over a one-year period) will lead to a severe under-utilization of the installation. Instead, if storage is considered as a service in the energy market, each power producer may rent the necessary capacity in each delivery period for minimizing power imbalances. Alternatively, one may use the dynamic sizing methodology introduced here for sizing storage given an accepted risk exposure to regulation costs.

The assessment of expected forecast uncertainty for each auctioning/delivery period is central for dynamic storage sizing. The methodology presented in this contribution shows how this decision can be made based on scenario forecasts derived from probabilistic forecasts and the most recent information on the interdependence structure of forecast errors for the set of forecast horizons of relevance. By assessing the combined wind/storage plant operation for all possible scenario forecasts over the next delivery period, a distribution for the necessary energy content may be obtained. When this distribution is obtained, the power producer may decide upon the desired risk exposure by choosing a storage capacity that corresponds to a specific quantile of this distribution. Note than in the future, more advanced information on expected regulation costs can be employed in this methodology, thus permitting to make more appropriate decisions on the sizing depending upon volatility of regulation unit costs. One could then evisage desingning optimal trading strategies accounting based on optimal use of storage capacity.

This proposal dynamic sizing methodology may be used as a basis for introducing energy storage as a market entity that is hedging the power producer against the risk due to the limited wind power predictability. The producer may estimate the necessary storage size and also decide on his risk exposure. The methodology can be used for assessing the profit and costs of implementing storage. Also, since the storage size is related to the forecast uncertainty, the dynamic sizing of energy storage is a flexible approach to anticipated improvement of forecast accuracy in the future. In market conditions that favor wind, the same methodology may be used from the side of the system operator, for the assessment of the necessary reserves for securing the system operation due to the uncertainty in wind power production. Finally, it should be mentioned that the methodology presented here is generic: dynamic storage sizing corresponds to the first application from a series of different tasks in the energy portfolio management that may be redefined accordingly, like reserve allocation, economic dispatch, etc., based on a stochastic optimization paradigm.

\section{ACKNOWLEDGEMENTS}

Vattenfall Denmark is acknowledged for providing the power data used as input.

\section{REFERENCES}

[1] H. Madsen, P. Pinson, T. S. Nielsen, H. A. Nielsen, and G. Kariniotakis, "Standardizing the performance evaluation of short-term wind power prediction models," Wind Engineering, vol. 29, no. 6, pp. 475-489, 2005.

[2] J. P. Barton and D. G. Infield, "Energy storage and its use with intermittent renewable energy," IEEE Transactions on Energy Conversion, vol. 19, no. 2, pp. 441-448, June 2004.

[3] — "A probabilistic method for calculating the usefulness of a store with finite energy capacity for smoothing electricity generation from wind and solar power," Journal of Power Sources, vol. 162, no. 2, pp. 943-948, 2006.

[4] M. Black and G. Strbac, "Value of bulk energy storage for managing wind power fluctuations," IEEE Transactions on Energy Conversion, vol. 22, no. 1, pp. 197-205, March 2007.

[5] J. S. Anagnostopoulos and D. E. Papantonis, "Simulation and size optimization of a pumped-storage power plant for the recovery of wind farms rejected energy," Renewable Energy, vol. 33, pp. 1685-1694, 2008.

[6] G. Koeppel and M. Korpaas, "Improving the network infeed accuracy of non-dispatchable generators with energy storage devices," Electric Power Systems Research, vol. 78, no. 12, pp. 2024-2036, August 2008.

[7] X. Y. Wang, D. M. Vilathgamuwa, and S. S. Choi, "Determination of battery storage capacity in energy buffer for wind farm," IEEE Transactions on Power Systems, vol. 23, no. 3, pp. 868-878, September 2008.

[8] P. Pinson, G. Papaefthymiou, B. Klöckl, H. A. Nielsen, and H. Madsen, "From probabilistic forecasts to statistical scenarios of short-term wind power production," Wind energy, vol. 12, no. 1, pp. 51-62, 2009.

[9] M. Lange, "On the uncertainty of wind power predictions - Analysis of the forecast accuracy and statistical distribution of errors," Journal of Solar Energy Engineering - Transactions of the ASME, vol. 127, pp. $177-184,2005$.

[10] P. Pinson, "Estimation of the uncertainty in wind power forecasting," Ph.D. dissertation, Ecole des Mines de Paris, Paris, France, 2006.

[11] K. Skytte, "The regulating power market on the nordic power exchange Nord Pool: An econometric analysis," Energy Economics, vol. 21, no. 4, pp. 295-308, August 1999. 
[12] P. Pinson, C. Chevallier, and G. N. Kariniotakis, "Trading wind generation from short-term probabilistic forecasts of wind power," IEEE Transactions on Power Systems, vol. 19, no. 3, pp. 1148-1156, August 2007.

[13] G. N. Bathurst, J. Weatherill, and G. Strbac, "Trading wind generation in short term energy markets," IEEE Transactions on Power Systems, vol. 17, no. 3, pp. 782-789, August 2002.

[14] J. Matevosyan and L. Söder, "Minimization of imbalance cost trading wind power on the short-term power market," IEEE Transactions on Power Systems, vol. 21, no. 3, pp. 1396-1404, August 2006.

[15] B. Klöckl, "Impacts of energy storage on power systems with stochastic generation," Ph.D. dissertation, ETH Zürich, Zürich, Switzerland, 2007.

[16] E. D. Castronuovo and J. A. Pecas-Lopes, "On the optimization of the daily operation of a wind-hydro power plant," IEEE Transactions on Power Systems, vol. 19, no. 3, pp. 1599-1606, August 2004.

[17] G. N. Bathurst and G. Strbac, "Value of combining energy storage and wind in short-term energy and balancing markets," Electric Power Systems Research, vol. 67, pp. 1-8, 2003.

[18] M. Korpaas, A. T. Holen, and R. Hildrum, "Operation and sizing of energy storage for wind power plants in a market system," Electrical Power and Energy Systems, vol. 25, pp. 599-606, 2003.

[19] J. K. Møller, H. A. Nielsen, and H. Madsen, "Time-adaptive quantile regression," Computational Statistics and Data Analysis, vol. 52, pp. 1292-1303, 2008.

[20] H. A. Nielsen, T. S. Nielsen, H. Madsen, J. Badger, G. Giebel, L. Landberg, K. Sattler, L. Voulund, and J. Tøfting, "From wind ensembles to probabilistic information about future wind power production results from an actual application," in 9th International Conference on Probabilistic Methods Applied to Power Systems (PMAPS), Stockholm, Sweden, June 11-15 2006.

[21] P. Pinson and H. Madsen, "Ensemble-based probabilistic forecasting at Horns Rev," Wind energy, Special issue on offshore wind energy, 2009.

[22] G. Papaefthymiou, "Integration of stochastic generation in power systems," Ph.D. dissertation, Power Systems Group, Delft University of Technology, Delft, The Netherlands, 2007.

[23] T. S. Nielsen, H. Madsen, and H. A. Nielsen, "Prediction of wind power using time-varying coefficient functions," in IFAC 2002, 'International Federation for Automatic Control', Barcelona, Spain, 2002.

[24] P. Pinson, H. A. Nielsen, J. K. Møller, H. Madsen, and G. Kariniotakis, "Nonparametric probabilistic forecasts of wind power: required properties and evaluation," Wind Energy, vol. 10, no. 6, pp. 497-516, 2007.

[25] U. Linnet, "Tools supporting wind energy trade in deregulated market," M.Sc. Thesis, Technical University of Denmark, Informatics and Mathematical modelling, Kgs. Lyngby, Denmark, 2005.

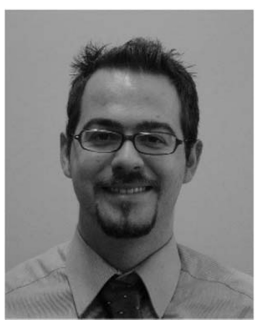

George Papaefthymiou (M'03) obtained his DiplEng in Electrical and Computer Engineering from the University of Patras, Greece in 1999 and the $\mathrm{Ph}$.D. degree from the Delft University of Technology, the Netherlands in 2007. He is currently with the Electrical Power Systems Laboratory of the Delft University of Technology, as a research associate and with Ecofys Germany Gmbh as a consultant. His current research interests include among others modeling of uncertainty in power systems and design of energy systems with large-scale penetration of distributed and stochastic generation.

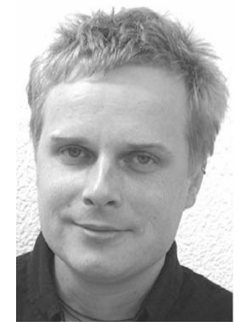

Bernd Klöckl (M’02) received the Dipl.-Ing. degree in Electrical Power Engineering from Graz University of Technology, Austria, in 2001 and the Ph.D. degree from ETH Zurich, Switzerland, in 2007, where he was research associate and lecturer from 2002 to 2006. From 2006 to 2007, he headed the grid section of the Austrian Association of Electricity Companies, Vienna, Austria. Since 2007, he is with the market management unit of the transmission system operator APG, responsible for cross-border tariffication and the development of observation methods for stochastic power flows in the transmission network. Dr. Klöckl is member of CIGRÉ and IEEE.

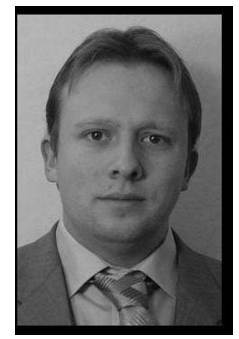

Jody Verboomen (S'03-M'05) received the M.I.S degree in Electronics from Groep T Hogeschool, Leuven, Belgium, in 2001 and the M.Sc. degree in Electrical Engineering from the Catholic University of Leuven (KUL) in 2004. He obtained his Ph.D. degree from the Electrical Power System (EPS) laboratory of the Delft University of Technology, Delft, The Netherlands in 2008. Currently, he is a consultant at Siemens AG, Erlangen, Germany.

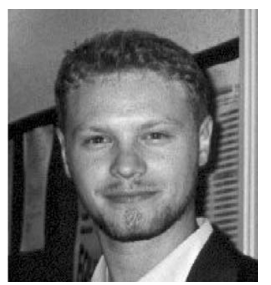

Pierre Pinson received the M.Sc. degree in Applied Mathematics from the National Institute for Applied Sciences (INSA Toulouse, France) in 2002 and the $\mathrm{Ph} . \mathrm{D}$. degree in Energetic from Ecole des Mines de Paris in 2006. He is currently with the Informatics and Mathematical Modeling department of the Technical University of Denmark as an Associate Professor. His research interests include among others forecasting, uncertainty estimation, optimization under uncertainty, decision sciences, and renewable energies. 